\title{
Use of a halobacterial bgaH reporter gene to analyse the regulation of gene expression in halophilic archaea
}

\author{
Dagmar Gregor and Felicitas Pfeifer
}

Institut für Mikrobiologie und Genetik, Technische Universität Darmstadt, Schnittspahnstr. 10, D-64287 Darmstadt, Germany
Author for correspondence: Felicitas Pfeifer. Tel: +49 6151 162957. Fax: +496151 162956. e-mail:pfeifer@bio.tu-darmstadt.de

The bgaH reading frame encoding a $\beta$-galactosidase of ' Haloferax alicantei' was used as a reporter gene to investigate three different promoter regions derived from gvpA genes of Haloferax mediterranei (mc-gvpA) and Halobacterium salinarum (c-gvpA and p-gvpA) in Haloferax volcanii transformants. The fusion of $b g a H$ at the start codon of each gvpA reading frame (A1-bgaH fusion genes) caused translational problems in some cases. Transformants containing constructs with fusions further downstream in the gvpA reading frame (A-bgaH) produced $\beta$-galactosidase, and colonies on agar plates turned blue when sprayed with X-Gal. The $\beta$-galactosidase activities quantified by standard ONPG assays correlated well with the mRNA data determined with transformants containing the respective gvpA genes: the cA-bgaH fusion gene was completely inactive, the mcA-bgaH transformants showed low amounts of products, whereas the pA-bgaH fusion gene was constitutively expressed in the respective transformants. The transcription of each A-bgaH gene was activated by the homologous transcriptional activator protein GvpE. The CGvpE, PGvpE and mcGvpE proteins were able to activate the promoter of pA-bgaH and mcA-bgaH, whereas the promoter of CA-bgaH was only activated by CGvpE. Among the three GvpE proteins tested, cGvpE appeared to be the strongest transcriptional activator.

Keywords: Haloferax volcanii, $\beta$-galactosidase reporter gene, gene regulation

\section{INTRODUCTION}

Halophilic archaea are model organisms to study archaeal gene regulation in vivo. They are easy to grow, and a transformation system including vector plasmids conferring resistance to mevinolin or novobiocin is available (Lam \& Doolittle, 1989; Holmes et al., 1991). Transcription in archaea possesses fundamental similarities to eukaryal transcription: archaeal promoters contain a TATA box element centred 25-28 nucleotides upstream of the transcription start site, and gene expression involves a single DNA-dependent RNA polymerase comprising 12 subunits that are homologous to the core components of the eukaryal RNA polymerase II (Zillig et al., 1993). Two additional factors, namely the TATA-box-binding protein TBP and TFB (the archaeal homologue of transcription factor TFIIB) are sufficient for the initiation of basal transcription (Hausner et al., 1996; Qureshi et al., 1995; Thomm,

Abbreviations: gvp, gas vesicle protein gene; Gvp, gas vesicle protein.
1996; Reeve et al., 1997). TFB recognizes a DNA sequence immediately upstream of the TATA box (the TFB recognition element BRE found in many archaeal genes) as demonstrated by in vitro transcription studies using recombinant factors and RNA polymerase from the thermophilic archaeon Sulfolobus (Qureshi \& Jackson, 1998; Bell et al., 1998, 1999). So far, in vitro transcription systems have been established for some methanogenic and thermophilic archaea, but not for halophilic archaea. The genome sequences of Methanococcus jannaschii and Methanobacterium thermautotrophicum contain single genes for TBP and TFB proteins, whereas halophilic archaea harbour multiple gene copies for both TBP and TFB (Ng et al., 1998; Baglia et al., 2000; Thompson et al., 1999).

We are using the genes encoding gas vesicles to study gene regulation in halophilic archaea. Gas vesicle formation involves the 14 genes gvpDEFGHIJKLM and gvpACNO that cluster in a genomic region called the vac region. Three different vac regions have been characterized, two of these are found in Halobacterium 
salinarum $\mathrm{PHH} 1$, namely the chromosomal c-vac region and the plasmid-borne p-vac region of the $150 \mathrm{~kb}$ plasmid pHH1 (Englert et al., 1992a). In the case of the related strain $H b$. salinarum NRC-1, two identical gvp1 gene clusters corresponding to the $\mathrm{p}$-vac region are present on the two large plasmids pNRC100 and pNRC200, and an additional gvp2 gene cluster almost identical to c-vac is found on pNRC200 (DasSarma et al., 1994; $\mathrm{Ng}$ et al., 1998, 2000). The mc-vac region (mediterranei chromosomal) is found in the chromosome of Haloferax mediterranei (Englert et al., 1992a). We use the designations c-gvp, p-gvp and mc-gvp to distinguish the various $g v p$ genes according to the origin of the vac region. The c-vac region is expressed only in p-vac deletion strains such as $\mathrm{Hb}$. salinarum $\mathrm{PHH} 4$ harbouring the $35 \mathrm{~kb}$ plasmid pHH4 (Pfeifer \& Blaseio, 1989).

The g $v p A C N O$ gene cluster encodes the major structural protein, GvpA, of the gas vesicles, and GvpC, a protein involved in stabilization of the structure and in shape formation (Offner et al., 1996). The products of the $g v p D$ and $g v p E$ genes are involved in regulation: GvpE is a transcriptional activator (Krüger \& Pfeifer, 1996; Krüger et al., 1998), whereas GvpD takes part in the repression of gas vesicle formation (Englert et al., 1992b; Pfeifer et al., 1994, 2001). These two Gvp proteins predominantly influence the promoter of the gvpACNO unit and thus determine whether and when gas vesicles are formed. The promoter of the p-gvpA gene of $\mathrm{Hb}$. salinarum PHH1 has a high activity throughout growth, and transcription results in large amounts of $\mathrm{p}$ - $q v p A$ mRNA plus minor amounts of $\mathrm{p}$ gvpACNO cotranscripts (Horne et al., 1991; Englert et al., 1992a). In contrast, the promoter of the c-gvpA gene is active only during the stationary phase in $\mathrm{Hb}$. salinarum PHH4 (Horne \& Pfeifer, 1989), similar to the promoter of the mc-gvpA gene of $H f$. mediterranei (Englert et al., 1992a).

The activities of the c-gvpA and mc-gvpA promoters depend on the presence of the GvpE activator protein, as demonstrated by transformation experiments using Haloferax volcanii as recipient. This strain does not contain the gvp genes and offers a clean genetic background. Transformants harbouring the p-gvpA gene produce p-gvpA mRNA throughout growth, whereas the respective c-gvpA and mc-gvpA transformants contain no or minor amounts of gvpA mRNA. However, transformants harbouring c-gvpA plus c-gvpE (expressed under $f d x$ promoter control) contain large amounts of c-gvpA mRNA and also GvpA protein (Krüger et al., 1998). Similarly, mc-gvpADE transformants produce large amounts of mc-gvpA transcripts and GvpA protein (Röder \& Pfeifer, 1996). These results demonstrate that GvpE is a transcriptional activator protein. Homology modelling of the C-terminal part of GvpE indicates an amphiphilic $\alpha$ helix that exhibits a similar structure as the one found in the basic leucine zipper (bZIP) activator protein GCN4 of yeast (Ellenberger et al., 1992; Krüger et al., 1998). A region adjacent to the $\mathrm{N}$ terminus consists of basic amino acids and constitutes a putative DNA-binding site. Such bZIP proteins are transcriptional activators predominantly found in eukarya.

To investigate the basal and induced activities of the three different gvpA promoters in $H f$. volcanii transformants in more detail, we tested a halobacterial $\beta$ galactosidase as reporter. The $b g a H$ gene encoding this enzyme has been isolated from a 'superblue' mutant of 'Haloferax alicantei' (Holmes et al., 1997; Holmes \& Dyall-Smith, 2000). Hf. volcanii lacks detectable $\beta$ galactosidase activity and is thus suitable for these studies. Transformants expressing the enzyme turn blue when sprayed with X-Gal on agar plates, and the $\beta$ galactosidase activity can be quantified by a standard assay hydrolysing ONPG. An application of this system for the investigation of various promoters in $H b$. salinarum has recently been reported by Patenge et al. (2000).

In this report, Hf. volcanii transformants were produced containing fusions between the various $g \nu p A$ promoter regions and the bgaH reading frame. Different fusion constructs were tested at the level of mRNA formation and at the level of $\beta$-galactosidase activity to gain insights into the amount of both products. $\beta$-Galactosidase activity did not always reflect the mRNA data, indicating that the fusion site is rather crucial for the translation of the bgaH reading frame. However, the A-bgaH fusion genes were sufficient to investigate $g v p A$ regulation.

\section{METHODS}

Growth conditions. Escherichia coli strains DH $5 \alpha$, XL-1 Blue (Stratagene) and GM1674, a dam-negative strain (Palmer \& Marinus, 1994) were grown aerobically at $37^{\circ} \mathrm{C}$ in LB broth (Sambrook et al., 1989). For selection of transformants, ampicillin was added at a concentration of $100 \mu \mathrm{g} \mathrm{ml}^{-1}$. Hf. volcanii WFD11, lacking the endogenous plasmid pHV2 (Cline et al., 1989), was grown in rich medium containing $\left(\mathrm{l}^{-1}\right): 175 \mathrm{~g} \mathrm{NaCl}, 37 \mathrm{~g} \mathrm{MgSO}_{4} .7 \mathrm{H}_{2} \mathrm{O}, 3.7 \mathrm{~g} \mathrm{KCl}, 5 \mathrm{~g}$ Bacto tryptone, $3 \mathrm{~g}$ Bacto yeast extract, $25 \mathrm{ml} 1 \mathrm{M}$ Tris/ $\mathrm{HCl} \mathrm{pH} \mathrm{7.2,}$ $5 \mathrm{ml} 10 \% \mathrm{CaCl}_{2} \cdot \mathrm{H}_{2} \mathrm{O}$ and $100 \mu \mathrm{l} 100 \mu \mathrm{M} \mathrm{MnCl} \mathrm{M}_{2}$. Transformants were selected on agar plates containing $0 \cdot 2 \mu \mathrm{g}$ novobiocin $\mathrm{ml}^{-1}$ and/or $6 \mu \mathrm{g}$ mevinolin $\mathrm{ml}^{-1}$. Lovastatin (a derivative of mevinolin) was a gift from MSD Sharpe \& Dohme.

Constructs used for transformation. The $2203 \mathrm{bp} \mathrm{bgaH}$ reading frame was amplified by PCR using the oligonucleotides bgaH-NcoI and bgaH-BamHI (Table 1), together with pMLH32 as template (Holmes \& Dyall-Smith, 2000). The NcoI site of primer $b g a H-N c o I$ included the ATG start codon of the bgaH reading frame, whereas the bgaH-BamHI primer was complementary to a sequence located $200 \mathrm{bp}$ downstream of the bgaH stop codon. Each gupA promoter region was amplified by PCR as a $\mathrm{XbaI}-\mathrm{NcoI}$ fragment using synthetic oligonucleotides (Table 1) and subfragments of the three different vac regions inserted in E. coli plasmids as templates. For the fusion at the ATG start codon of $g v p A$, the respective A-XbaI primer was used, together with pA1-NcoI (amplification of the $109 \mathrm{bp}$ pA1 promoter fragment), cA1NcoI (109 bp cA1 promoter fragment), and mcA1-NcoI (119 bp mcA1 promoter fragment) (see Fig. 1). Slightly larger 
Table 1. Synthetic oligonucleotides used for the amplification by PCR

\begin{tabular}{|llc|}
\hline Primer & \multicolumn{1}{c|}{ Sequence* } & Position $\dagger$ \\
\hline bgaH-NcoI & CATTGTCCATGGCAGTTGGTGTCTG & $2356-2380^{a}$ \\
bgaH-BamHI & GTGACGCGGATCCGCGTGTGTAC & $4571-4549^{a}$ \\
cA-X $b a \mathrm{I}$ & CTTGTTCTAGATTTTGTAC & $6087-6117^{b}$ \\
cA1-NcoI & CCGCCAAGCCCATGGAGTCTGG & $6237-6213^{b}$ \\
cA-NcoI & GTTGCGCCATGGTGATCTGG & $6216-6197^{b}$ \\
pA-XbaI & CTCCGTATCTAGAAGTACGAC & $13-33^{a}$ \\
pA1-NcoI & CTGCCAAGCCCATGGAATCTGGTTG & $155-131^{a}$ \\
pA-NcoI & GGTTGCGCCATGGGTGATCTG & $135-115^{a}$ \\
mcA-XbaI & CCAAACTATCTAGATGTTTGAC & $4213-4192^{c}$ \\
mcA1-NcoI & CCGCCAGACCCATGGAATCGGGC & $4070-4092^{c}$ \\
mcA-NcoI & TGAACCATGGTTGATCTGGG & $4093-4112^{c}$ \\
pE1 & CGGAGATGGTGGATCCATGGACGACTTGC & $1690-1708^{e}$ \\
pE2 & GATCTTCCTGTCTGCAGGTACCGTATGTGTATAGG & $2309-2275^{e}$ \\
mcE1 & CGGAGATGGTGTGACCCATGGAAC & $5936-5959^{c}$ \\
mcE2 & GCCGTAAGTGTACAGATTATCGC & $6547-6525^{c}$ \\
pAcA & CCGGAAAACGATGTGTATGGTTTCACCAGTCGTTTCGGC & $6167-6127^{b}$ \\
\hline
\end{tabular}

* Sequences underlined or in italic indicate restriction sites.

† $a$, bgaH sequence; $b$, c-vac sequence; $c$, mc-vac sequence; $d$, p-gvpACNO sequence; $e, \mathrm{p}$-gvpD-M sequence.

fragments spanning the ATG start codon of gvpA were amplified using the respective A-XbaI primer together with $\mathrm{pA}-\mathrm{NcoI}$ (resulting in the $127 \mathrm{bp}$ pA promoter fragment), cANcoI (137 bp cA promoter fragment) and mcA-NcoI (126 bp $\mathrm{mcA}$ promoter fragment). For the chimeric pAcA promoter fragment, a $60 \mathrm{nt}$ megaprimer was amplified using the primers $c A-X b a I$ and pAcA and the c-gvpA gene as template. The resulting megaprimer included $21 \mathrm{bp}$ of the $\mathrm{p}-g \nu p A$ promoter sequence fused to the TATA box of the c-gvpA gene; this megaprimer was used together with primer cA-NcoI in the second PCR to amplify the entire pAcA promoter fragment.

Each promoter fragment was purified by agarose gel electrophoresis, cleaved with $\mathrm{XbaI} / \mathrm{NcoI}$, and ligated with the $b g a H$ reading frame (cleaved with $\mathrm{NcoI}$ and BamHI) and the $X b a \mathrm{I} /$ BamHI-hydrolysed E. coli vector pBluescript II SK(+) (Stratagene). The exact fusion in each A1-bgaH and A-bgaH gene was confirmed by DNA sequence analysis. The A1-bgaH and $\mathrm{A}-b g a H$ genes were transferred as $\mathrm{XbaI} / \mathrm{BamHI}$ fragments into the halobacterial vector plasmid pWL102 (Lam \& Doolittle, 1989). For the expression of the p-gvpE and mcgvpE reading frames, both were amplified by PCR using the primer pairs $\mathrm{pE} 1 / \mathrm{pE} 2$, or $\mathrm{mcE} 1 / \mathrm{mcE} 2$ (Table 1 ). The PCR products were inserted into the halobacterial expression vector pJAS35 for expression under $f d x$ promoter control (Pfeifer et al., 1994). The c-gvpE-pJAS construct has already been described (Krüger et al., 1998).

Transformation of Hf. volcanii WFD11. Prior to the transformation of $H f$. volcanii, each construct was passaged through E. coli GM1674 (dam-negative) to avoid a halobacterial restriction barrier (Holmes et al., 1991). Transformation was carried out as described previously (Pfeifer \& Ghahraman, 1993). The presence and the amount of the desired plasmid(s) in each transformant was determined by Southern analyses using specific DNA probes. A $3.5 \mathrm{~kb}$ HindIII/BamHI fragment derived from pMLH32 was used for bgaH, whereas 600-700 bp Acc65I/NcoI fragments containing the respective $g v p E$ reading frame were used to generate the gvpE-specific probes. Each probe was labelled with digoxigenin using the DIG-Labelling Kit from Roche.

RNA isolation and transcript analysis. Total RNA was isolated by the method of Chomczynski \& Sacchi (1987), or by using the RNeasy Kit from Qiagen, followed by DNase I digestion. For transcript analysis, 5 or $10 \mu \mathrm{g}$ RNA was separated on denaturing, formaldehyde-containing $1.2 \% \quad(\mathrm{w} / \mathrm{v})$ agarose gels, followed by transfer to nylon membranes (Ausubel et al., 1988). A strand-specific bgaH RNA probe was synthesized with the T3/T7 system using the $2 \cdot 2 \mathrm{~kb}$ NcoI-BamHI bgaH fragment inserted in pBluescript as template. The probe was labelled with digoxigenin using the DIG RNA Labelling Kit obtained from Roche.

$\boldsymbol{\beta}$-Galactosidase assay. $\beta$-Galactosidase activity in colonies was visualized by spraying the transformant colonies grown on agar plates with X-Gal $\left(10 \mathrm{mg} \mathrm{ml}^{-1}\right)$. $\beta$-Galactosidase activity in cell lysates was measured using the ONPG assay as described by Holmes et al. (1997). The protein concentration was determined by the Bradford assay (Ausubel et al., 1988) using BSA as standard.

Accession numbers of DNA sequences. These are as follows: U70664 (bgaH gene), X94688 (c-vac region of $\mathrm{Hb}$. salinarum PHH4), X64701 (mc-vac region of Hf. mediterranei), X64729 (p-gvpACNO) and X55648 (p-gvpDEFGHIJKLM). The latter two sequences derive from the $\mathrm{p}$-vac region on plasmid $\mathrm{pHH} 1$ of Hb. salinarum PHH1 (formerly Hb. halobium NRC817).

\section{RESULTS}

Three different gvpA genes encoding the major gas vesicle structural protein of $H b$. salinarum and $H f$. mediterrane $i$ were used for a comparative investigation of gene expression. These genes are transcribed as $340 \mathrm{nt}$ mRNAs containing a 19-20 nt leader region (Horne \& Pfeifer, 1989; Englert et al., 1990). The gvpA mRNA leader regions are almost identical except for a $4 \mathrm{nt}$ sequence at position 5-8 of the mRNA, and the 
(a)

cA AAACGGGGGTTGAACTCACAACGGCGGTTTTCCGGACACTCCCTGTAGTTGCGGGTGGGTACCACCCAGATCACTATGG

pAcA AAACGACTGGTGAAACCATACACATCGTTTTCCGGACACTCCCTGTAGTTGCGGGTGGGTACCACCCAGATCACTATGG

$\mathrm{pA}$ TAACGACTGGTGAAACCATACACATCCTTATGTGATGCCCGAGTATAGTTAGAGATGGGTTAATCCCAGATCACCAATGG $\mathrm{mcA}$ AAACGGTTGCTGAACCAACACGAATGATTTTGTTACTTGCCAACACGTTTTCAGATGGGTATAACCCAGATCAATCATGG

(b)

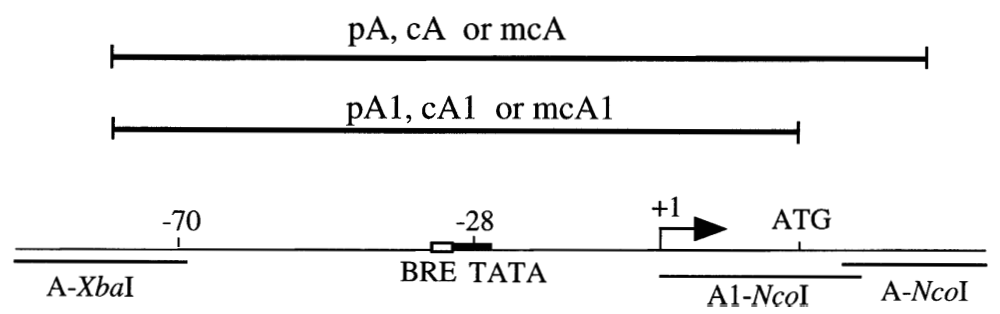

\begin{abstract}
Fig. 1. (a) Promoter region of the three $g v p A$ genes and of the $p A c A$ hybrid, and (b) extent of the $A 1$ - and A-promoter fragments. (a) The start site of transcription (G) is labelled +1 , and the ATG start codon is shown in bold. The TATA box centred around -28 is shown in bold. The consensus sequences of the BRE element and of the TATA box are given above $(\mathrm{R}=\mathrm{A}$ or $\mathrm{G}, \mathrm{N}=$ any base, $\mathrm{W}=\mathrm{A}$ or $\mathrm{T}, \mathrm{Y}=\mathrm{C}$ or $\mathrm{T})$. Sequences derived from the $\mathrm{p}$-gvpA promoter in the $\mathrm{pAcA}-b g a H$ promoter are underlined. The nucleotide sequences around the ATG start codon used to introduce the Ncol site (CCATGG) during the construction of the A1-bgaH gene fusions are shown in italic. (b) Schematic representation of the gvpA promoter region. including the TATA box and the putative BRE element as well as the start sites of transcription $(+1)$ and of translation (ATG). The bars below the map indicate the various oligonucleotides used for the PCR amplifications. The lines above the map represent the promoter fragments $p A 1, C A 1$ and mcA1, or the slightly larger fragments $\mathrm{pA}, \mathrm{CA}$ and $\mathrm{mcA}$.
\end{abstract}

nucleotides adjacent to the ATG start codon (Fig. 1a). No sequences reminiscent of a Shine-Dalgarno motif are present. The mRNA of the bgaH gene of ' $H f$. alicantei' also contains a 34 nt leader region with no obvious Shine-Dalgarno motif (Holmes \& Dyall-Smith, 2000).

To investigate the regulatory elements that contribute to the expression of the gvpA genes in Hf. volcanii transformants in more detail, we amplified fragments containing the respective gvpA promoter regions, including the region transcribed into the mRNA leader (Fig. 1b). The first round of amplifications resulted in the fragments pA1, cA1 and mcA1 (109-119 bp) containing the ATG start codon of the respective g $v p A$ gene within the NcoI cloning site. The second series of amplifications resulted in the 15-nt-larger $\mathrm{pA}, \mathrm{cA}$ and mcA fragments (see Fig. 1b). The 2203 bp bgaH reading frame, amplified as a NcoI/BamHI fragment, was fused to the respective A1- and A-promoter fragments at the ATG start codon of $b g a H$. The A1-bgaH fusions encoded the native $\beta$-galactosidase, whereas the $\mathrm{A}-b g a H$ fusions encoded a $\beta$-galactosidase protein with five additional amino acids derived from GvpA at the $\mathrm{N}$ terminus (MAQPD or MVQPD, see below). Constructs containing the $\mathrm{A} 1-b g a H$ or $\mathrm{A}-b g a H$ fusion genes were used to transform Hf. volcanii. Transformants containing only the A-bgaH (or A1-bgaH) construct were used to monitor the basal amount of mRNA and $\beta$ - galactosidase activity, whereas transformants harbouring in addition one of the three different $g v p E$ pJAS constructs (i.e. A-bgaH/E or A1-bgaH/E transformants) were used to determine the GvpE-dependent activity of each $g \nu p A$ regulatory region.

\section{Reporter gene fusion at the ATG start codon of the gvpA reading frame}

The various A1-bgaH and A1-bgaH/E transformants were analysed by Southern blotting for the presence of the desired construct(s). The desired transformants were streaked as cross-shaped colonies on agar plates and sprayed with X-Gal solution after growth to demonstrate $\beta$-galactosidase activity (Fig. 2). Colonies of $H f$. volcanii are usually orange-red and retain this colour after X-Gal treatment, similar to Hf. volcanii containing the c-gvpE gene (cE, Fig. 2). The transformants containing the original bgaH construct pMLH32 (Holmes \& Dyall-Smith, 2000) turned dark blue (bgaH, Fig. 2). Transformants containing the mcA1-bgaH construct (mcA1) formed brownish red colonies, consistent with the very low activity of the mc-gvpA promoter in transformants harbouring the mc-gvpA gene (Röder \& Pfeifer, 1996). The mcA1-bgaH/cE transformants (mcA1-bgaH plus c-gvpE-pJAS) turned dark blue, demonstrating the induction of mcA1-bgaH expression by the $\mathrm{cGvpE}$ protein $(\mathrm{mcA} 1 / \mathrm{cE}, \mathrm{Fig}$. 2). The cA1-bgaH transformants (cA1) remained orange-red, which is 


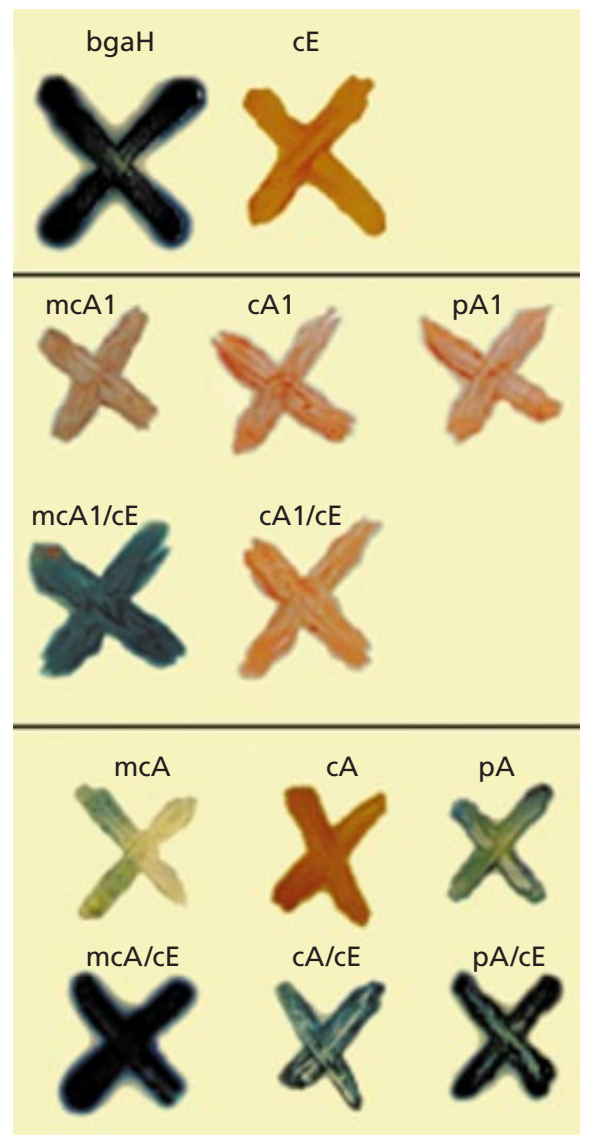

Fig. 2. Colonies of Hf. volcanii transformants sprayed with $\mathrm{X}$ Gal. The cells were grown as cross-shaped colonies on agar medium and sprayed with $10 \mathrm{mg} \mathrm{X}-\mathrm{Gal} \mathrm{ml}^{-1}$. The constructs present in each transformant are indicated above the colonies. The designation ' $\mathrm{bgaH}^{\prime}$ ' indicates the presence of plasmid pMLH32, and ' $c E$ ' indicates construct c-gvpE-pJAS. The respective $\mathrm{A} 1-b g a H$ and $A-b g a H$ fusion genes are inserted in pWL102. The origin of the promoter in the A1- and A-bgaH fusion genes is indicated by ' $\mathrm{mc}$ ' (mediterranei chromosomal), ' $c$ ' (c-vac) and ' $p$ ' ( $p$-vac).

indicative of the inactive c-gvpA promoter, similiar to the complete lack of detectable amounts of c-gvpA mRNA in transformants containing the c-gvpA gene (Krüger \& Pfeifer, 1996). However, colonies of the cA1-bgaH/cE transformants (cA1/cE) also remained orange-red, demonstrating that these cells did not contain detectable amounts of $\beta$-galactosidase activity (Fig. 2). This result was in contrast to the data obtained with c- $g v p A+c-g v p E-p J A S$ transformants which produce large amounts of c-gvpA mRNA and GvpA protein (Krüger et al., 1998). Also, transformants containing the pA1-bgaH construct (pA1) remained red although the p-gvpA gene is transcribed in p-gvpA transformants. Since the cA1-bgaH and pA1-bgaH genes contained the entire promoter and regulatory region, including the transcriptional start site of the respective g $v p A$ gene, these results raised the question of whether the lack of $\beta$ galactosidase activity was caused by transcriptional or translational problems.

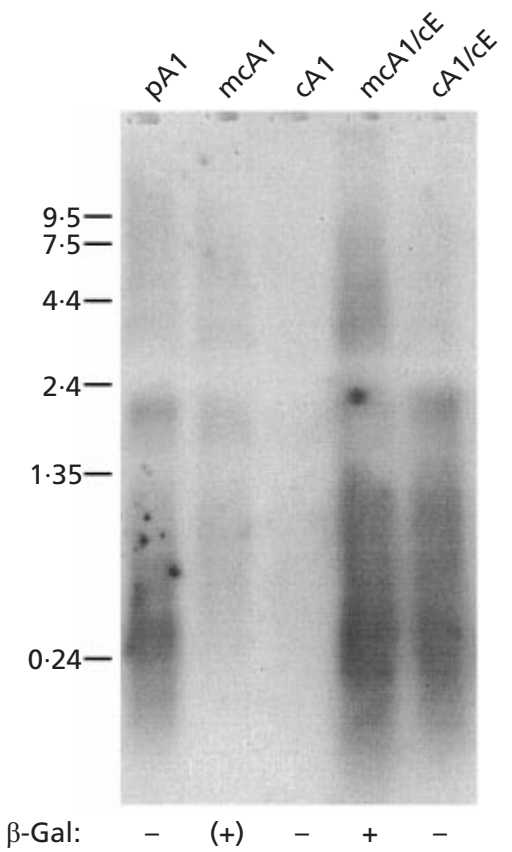

Fig. 3. Northern analysis to determine the presence of bgaH transcripts. RNA samples derived from exponential growth of the pA1-, mcA1- and cA1-bgaH, and mcA1-bgaH/cE and cA1-bgaH/cE transformants were separated on $1.2 \%$ agarose gels and hybridized with the $2 \cdot 2 \mathrm{~kb} \mathrm{Ncol-BamHI} \mathrm{bgaH}$ fragment as strand-specific probe. The RNA marker sizes (in $\mathrm{kb}$ ) are given on the left. $\beta$-Galactosidase activity was also determined as indicated below the autoradiogram:,$-(+)$ and + indicate the intensity of the blue colour (see Fig. 2).

Northern analyses were performed to monitor the A1-bgaH transcriptional levels. Total RNA was isolated from the transformants, electrophoretically separated on an agarose gel and hybridized with a bgaH-specific probe (Fig. 3). The $2 \cdot 8 \mathrm{~kb}$ bgaH mRNA (and degradation products) was detected in pA1-bgaH and mcA1bgaH transformants, whereas cA1-bgaH transformants did not contain detectable amounts of bgaH transcript. These results correlated with the earlier mRNA studies on the respective gvpA transformants. The mcA1$b g a H / c E$ and cA1-bgaH/cE transformants contained large amounts of $b g a H$ mRNA, demonstrating that the transcription of both A1-bgaH genes was activated by cGvpE. Since the transcript formation in all of these transformants was as expected, the lack of $\beta$-galactosidase activity in case of the pA1 and $\mathrm{cA} 1 / \mathrm{cE}$ transformants was likely due to a problem that occurred at the level of translation.

\section{Reporter gene fusions within the gvpA reading frame}

The second series of fusion genes $(\mathrm{A}-b g a H)$ contained the $b g a H$ reading frame fused to the larger $\mathrm{pA}, \mathrm{cA}$ or mcA promoter fragments. The fusion was generated in the $g v p A$ reading frame, resulting in $\beta$-galactosidase proteins containing five amino acids of GvpA (MAQPD 


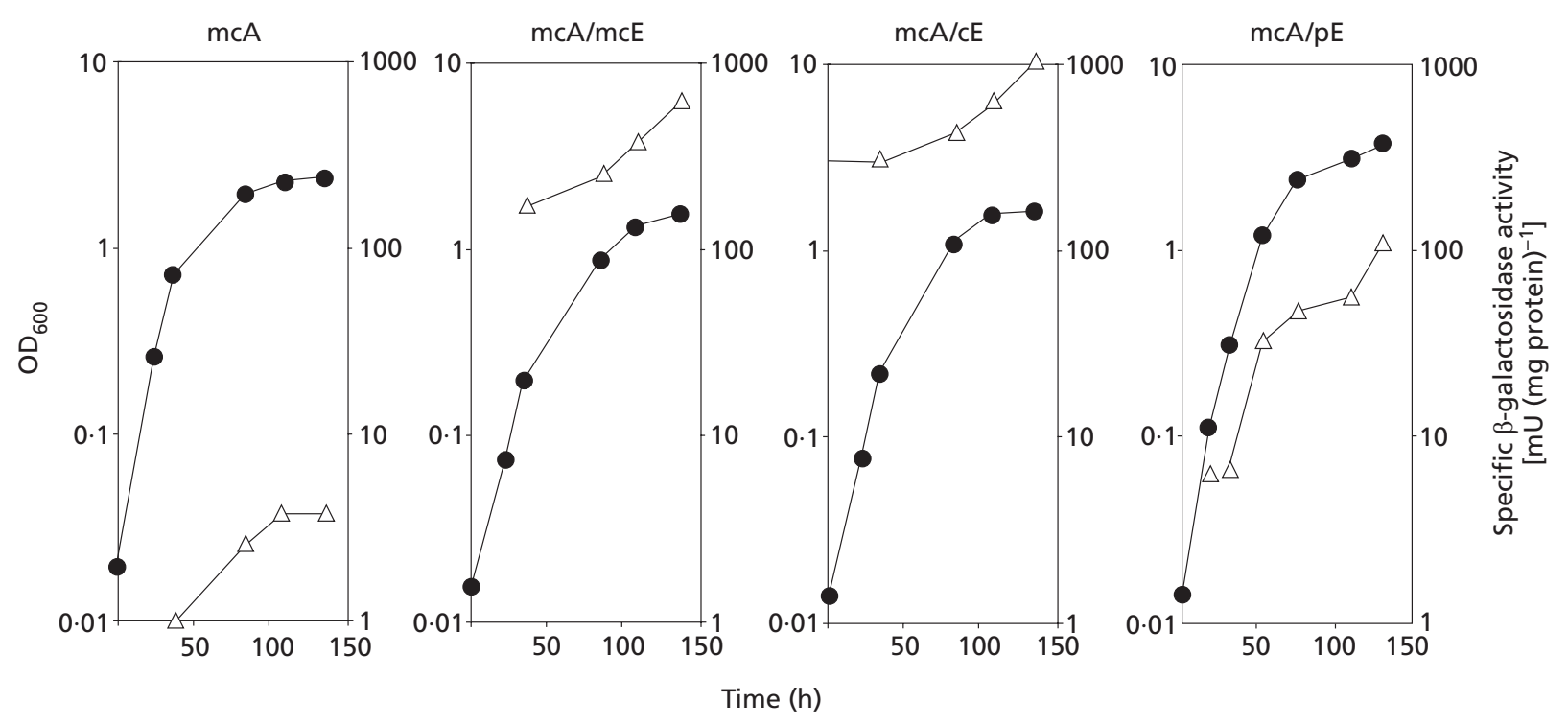

Fig. 4. Growth curves and $\beta$-galactosidase activities determined for mcA-bgaH and various mcA-bgaH/E transformants. Each plot shows the growth curve $(\bullet)$ and the specific $\beta$-galactosidase activity $(\triangle)$ of transformants containing the mcA-bgaH gene (mcA), or mcA plus mc-gvpE (mcE), c-gvpE (cE) or p-gvpE (pE) in pJAS35. $\beta$-Galactosidase activities were determined by the ONPG assay.

in the case of the p-gvpA and c-gvpA, and MVQPD in the case of the mc-gvpA fusion). Transformants contained either the A-bgaH construct by itself (to determine the basal product levels), or together with one of the three gvpE-pJAS constructs. All transformants were analysed by Southern blotting for the presence of the desired plasmids. Colonies of each of these transformants were sprayed with X-Gal solution for the detection of $\beta$-galactosidase activity (Fig. 2, bottom). The mcA-bgaH transformants (mcA) were light blue, presumably due to low $\beta$-galactosidase production, the $\mathrm{cA}-b g a H$ transformants $(\mathrm{cA})$ remained orange-red (no $\beta$-galactosidase production), and the $\mathrm{pA}-b g a H$ transformants $(\mathrm{pA})$ were blue due to a higher amount of $\beta$ galactosidase activity. Each transformant containing one of the A-bgaH genes plus c-gvpE-pJAS showed a significant increase in blue colour: the activation was strongest with the mcA-bgaH/cE transformant (mcA/ $\mathrm{cE})$, but also the cA-bgaH/cE $(\mathrm{cA} / \mathrm{cE})$ and the pA$b g a H / c E$ transformants $(\mathrm{pA} / \mathrm{cE}$ ) turned dark blue (Fig. 2 , bottom). Thus, the A-bgaH fusion genes revealed $\beta$ galactosidase activities according to the gvpA mRNA levels in the respective $g v p A+\mathrm{c}-g v p E$ (or + mc-gvpE) transformants (Röder \& Pfeifer, 1996; Krüger et al., 1998).

The expression of the different A-bgaH genes in transformants was investigated with each of the three GvpE proteins, and the specific $\beta$-galactosidase activities were quantified in samples taken throughout the growth period using the ONPG assay. The analyses of the transformants harbouring the mcA-bgaH construct by itself or including one of the three gvpE genes $(\mathrm{mcA}$, $\mathrm{mcA} / \mathrm{pE}, \mathrm{mcA} / \mathrm{cE}$, and $\mathrm{mcA} / \mathrm{mcE}$ transformants) is presented in Fig. 4; similar experiments were done with all other transformants (data not shown). Since the $\beta$ galactosidase activities increased during exponential and early stationary phases, a mean activity was calculated from the activities determined in samples taken between 100 and $150 \mathrm{~h}$ of growth (Table 2). The highest specific $\beta$-galactosidase activity determined among the noninduced $\mathrm{A}-b g a H$ genes was found with $\mathrm{pA}-b g a H$ transformants; mcA-bgaH transformants showed a low activity, whereas cA-bgaH transformants did not contain detectable $\beta$-galactosidase activity (Table 2). The activation of each A-bgaH gene by GvpE was tested in the three different A-bgaH/E transformants. The various $\mathrm{mcA}-b g a H / \mathrm{E}$ transformants demonstrated high $\beta$-galactosidase activities (ranging from 120 to $900 \mathrm{mU} \mathrm{mg}^{-1}$ ), with pGvpE as the weakest and cGvpE as the strongest activator protein (Table 2). The pA-bgaH gene was also activated by all three GvpE proteins, but the expression of cA-bgaH was only achieved with the homologous cGvpE protein (cAbgaH/cE transformants, Table 2). The specific $\beta$ galactosidase activity determined in the latter transformant was slightly higher than the basal $\beta$-galactosidase activity in the pA-bgaH transformants. None of the heterologous GvpE activator proteins was able to activate the cA-bgaH gene (Table 2). In each case, the cGvpE protein appeared to be the strongest transcriptional activator protein among the three GvpE proteins (Table 2).

\section{Amount of bgaH mRNA and $\beta$-galactosidase activity throughout growth}

The different A-bgaH and respective A-bgaH/cE transformants were investigated at the mRNA level for the expression of the A-bgaH gene. The cells were grown in 
Table 2. Specific $\beta$-galactosidase activities of the various transformants

Values are means of samples taken between 100 and $150 \mathrm{~h}$ of growth. ND, Not detectable.

\begin{tabular}{|c|c|c|c|c|}
\hline \multirow[t]{2}{*}{ Transformant } & \multicolumn{4}{|c|}{ Specific $\beta$-galactosidase activity $\left[\mathrm{mU}(\mathrm{mg} \text { protein })^{-1}\right]$} \\
\hline & Basal & $+\mathrm{p}-g v p E$ & $+\mathrm{mc}-g v p E$ & $+c-g v p E$ \\
\hline $\mathrm{mcA}-b g a H$ & 6 & 120 & 480 & 900 \\
\hline $\mathrm{pA}-b g a H$ & 13 & 200 & 170 & 240 \\
\hline$c A-b g a H$ & ND & ND & ND & 20 \\
\hline pAcA-bgaH & ND & 4 & 15 & 65 \\
\hline
\end{tabular}

(a)

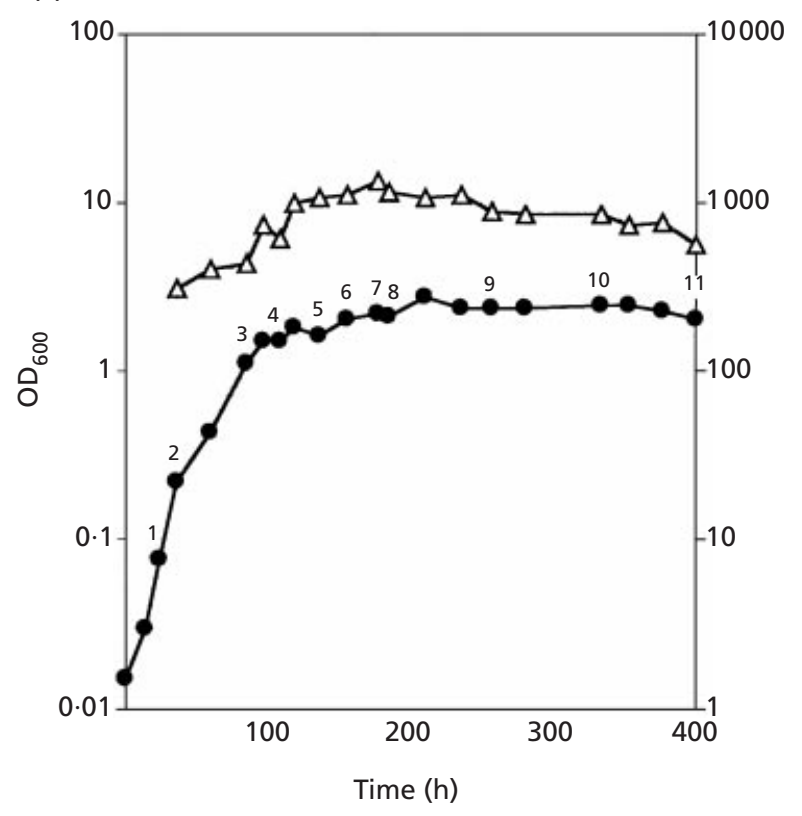

(b)

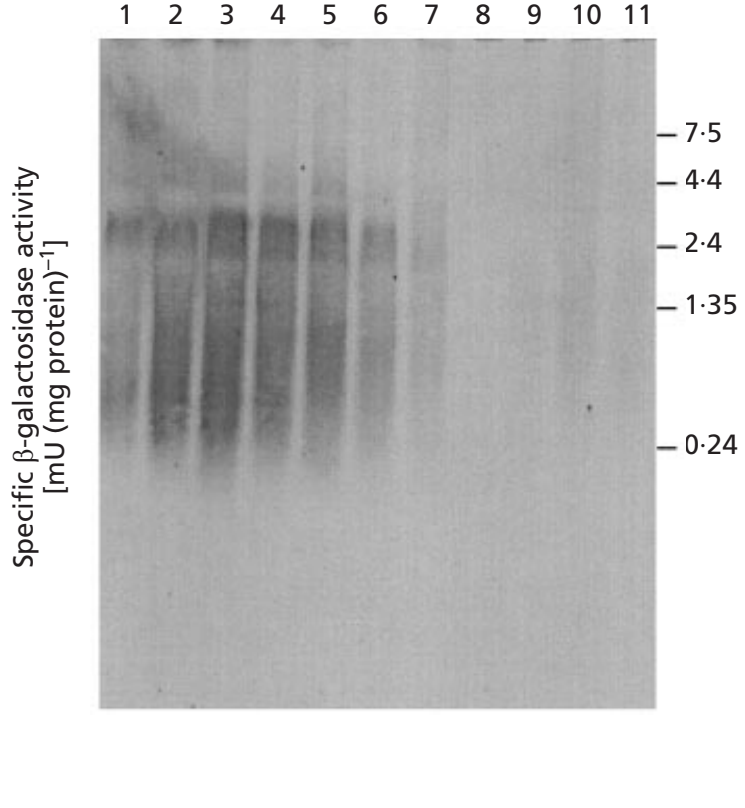

Fig. 5. (a) Growth curve and $\beta$-galactosidase activities, and (b) Northern analysis to detect bgaH mRNA in the mcA-bgaH/cE transformant. (a) Samples were taken during growth $(\bullet)$, and the specific $\beta$-galactosidase activities ( $\triangle$ ) determined by the ONPG assay. The numbers above the growth curve indicate samples used for Northern analysis. (b) RNA isolated from samples 1-11 was used for Northern analysis and hybridized with a digoxigenin-labelled, bgaH-specific probe. RNA marker sizes (in kb) are given on the right.

$25 \%$ salt medium, and samples were taken during the exponential and stationary phases for RNA isolation and Northern analysis. The $2 \cdot 8 \mathrm{~kb}$ bgaH mRNA (and degradation products) was detected in all transformants (data not shown). The mcA-bgaH/cE transformant was used to compare the amount of bgaH mRNA produced during growth with the $\beta$-galactosidase activities measured in the respective cell lysates. Eleven samples were taken throughout growth in $25 \%$ salt medium, and RNA was isolated and hybridized with the $b g a H$-specific probe (Fig. 5). The bgaH mRNA was mainly detected in samples derived from exponential and early stationary phases (Fig. 5b, lanes 1-7). Only minimal amounts of bgaH mRNA were detectable after 185 h (Fig. 5b, lanes $8-11)$. The $\beta$-galactosidase activity was also determined for each sample and indicated an increase during the exponential and the early stationary phase (up to $150 \mathrm{~h}$ ), which paralleled the increase in the bgaH mRNA (Fig. 5a). However, despite the minor amounts of $b g a H$ mRNA observed during the stationary phase, the $\beta$ galactosidase activities remained constantly high, indicating that the $\beta$-galactosidase protein was rather stable and remained active.

\section{pAcA promoter to investigate the region required for GvpE activation}

A chimeric $g v p A$ promoter was constructed by substituting a region of $21 \mathrm{nt} 5^{\prime}$ to the TATA box of the cA promoter with the respective $21 \mathrm{nt}$ derived from the $\mathrm{pA}$ promoter (see Fig. 1a) and the resulting pAcA-bgaH gene was tested for $\beta$-galactosidase activities and poss- 
ible activation by the various GvpE proteins in transformants. Transformants containing the pAcA-bgaH construct did not produce detectable amounts of $\beta$ galactosidase activity, similar to transformants containing the cA-bgaH construct (Table 2). However, the transformants harbouring the pAcA-bgaH construct plus one of the three gvpE-pJAS constructs produced $\beta$ galactosidase, with pAcA-bgaH/pE exhibiting the lowest and pAcA-bgaH/cE the highest $\beta$-galactosidase activity (Table 2). Compared to the transformants containing the original $\mathrm{pA}-$ and $\mathrm{cA}-b g a H$ sequences, a threefold higher $\beta$-galactosidase activity was found compared to cA-bgaH/cE, but the pAcA-bgaH/cE transformant attained only $36 \%$ of the $\beta$-galactosidase activity of the pA-bgaH/cE transformant (Table 2). Thus, the pAcA promoter acquired the ability for activation by the heterologous GvpE activator proteins.

\section{DISCUSSION}

The promoter of the plasmid-encoded p-gvpA gene of $H b$. salinarum PHH1 is highly active, whereas the respective c-gvpA and mc-gvpA promoters depend on the activation mediated by the GvpE activator proteins (Röder \& Pfeifer, 1996; Krüger et al., 1998). The bgaH reading frame encoding the $\beta$-galactosidase of ' $H f$. alicantei' (Holmes \& Dyall-Smith, 2000) was used to investigate the basal and induced activities of the various $g v p A$ promoter regions in $H f$. volcanii transformants. Different fusion sites between the promoter fragments and $b g a H$ were tested. One series of constructs contained the $b g a H$ reading frame fused directly at the ATG start codon of the respective g $v p A$ gene (pA1-, cA1- and mcA1-bgaH), whereas the second series (pA-, cA- and mcA-bgaH) contained the fusion site further downstream, resulting in $\beta$-galactosidase proteins containing five additional amino acids of GvpA at the $\mathrm{N}$ terminus.

\section{Reporter gene fusions at the ATG start codon of the gvpA gene may lead to problems in translation}

All A1-bgaH and A1-bgaH/cE transformants contained the expected amount of $b g a H$ mRNA. The mcA1-bgaH, cA1-bgaH and mcA1-bgaH/cE transformants also showed $\beta$-galactosidase activity, indicating that the mRNA consisting of the $g v p A$ mRNA leader and the $b g a H$ reading frame was indeed translated. However, the pA1-bgaH and cA1-bgaH/cE transformants did not show detectable amounts of $\beta$ galactosidase activity despite the presence of the mRNA, suggesting that a problem occurred at the translational level. A single point mutation ( $\mathrm{T}$ or $\mathrm{A} \rightarrow \mathrm{C}$ ) was introduced $5^{\prime}$ to the ATG start codon during the construction of the NcoI site for the fusion (Fig. 1a). Presumably, this alteration must be the reason for the lack of translation of these pA1- and cA1-bgaH mRNAs. However, a similar alteration was introduced in the mcA1-bgaH mRNA without preventing $\beta$ galactosidase formation. The leader sequences of the gvpA mRNAs are similar but not identical, and it is still unclear which regions are important for the initiation of translation. It is possible that the mutations destroyed a signal important for translation initiation, or forced an mRNA secondary structure that masked the AUG start codon. Various stem-loop secondary structures are possible in the leader region that are currently under investigation. Regardless, these results imply that the fusion of the $b g a H$ reporter gene should not be made at the start codon of the gene under investigation.

\section{The $\beta$-galactosidase activity reflects the activity of the gvpA promoters}

The larger promoter fragments used for the $\mathrm{pA}-, \mathrm{cA}-$ and mcA-bgaH constructs revealed the expected mRNAs that, when present, were translated into $\beta$ galactosidase. The analysis of the product formation in each $\mathrm{A}-b g a H$ transformant indicated no mRNA and $\beta$ galactosidase in the cA-bgaH transformant, a low mRNA amount and $\beta$-galactosidase activity in the mcA-bgaH, and a high mRNA amount and $\beta$-galactosidase activity in the pA-bgaH transformant. These results reflected the activities of the various $g \nu p A$ promoters in gvpA transformants (Röder \& Pfeifer, 1996; Krüger et al., 1998). Each promoter of the A-bgaH genes was stimulated at least by the homologous GvpE protein, indicating that the binding site of GvpE is contained within the promoter fragments used for the construction. The promoter of the mc-gvpA gene was the strongest activated promoter tested. Although the basal $\beta$-galactosidase activity was low in $\mathrm{mcA}-b g a H$ transformants, the activity was stimulated 80 -fold by the homologous mcGvpE protein and 150-fold by the cGvpE protein. Among the various GvpE proteins tested with the mc-gvpA promoter, pGvpE appeared to be the weakest and cGvpE the strongest activator. The $\mathrm{pA}-$ bgaH construct was also stimulated by all three GvpE proteins in respective $\mathrm{pA}-b g a H / \mathrm{E}$ transformants, but the overall induction was lower, except for the homologous pGvpE protein. Also, in this case, cGvpE appeared to be the strongest activator. It is possible that the single amino acid difference in the GvpA sequence near the $\mathrm{N}$ terminus results in a difference in the specific activity of the two $\beta$-galactosidase proteins (MVQPD in the case of mcA-bgaH and MAQPD in the case of cAand $\mathrm{pA}-b g a H)$. However, the products of the $\mathrm{pA}-b g a H$ and the cA-bgaH genes are identical, and at least these specific $\beta$-galactosidase activities can be compared with each other.

Similar to the c-gvpA gene, the cA-bgaH fusion gene was completely inactive in transformants. Only the homologous cGvpE activator was able to induce mRNA and $\beta$-galactosidase formation, but the activity remained relatively low. The pGvpE and mcGvpE proteins were unable to induce the c-gvpA promoter, which could be due to a weaker activity, but the DNA sequence responsible for GvpE binding could also contribute. The inability of $\mathrm{pGvpE}$ to activate the c-gvpA promoter has already been recognized during heterologous complementation studies using the p-gvpDEFGHIJKLM unit of the p-vac region to complement the c-gvpACNO genes in $H f$. volcanii transformants: no GvpA formation could be observed, suggesting that pGvpE cannot 
activate the c-gvpA promoter (Offner et al., 1998). This could also be the reason that gas vesicles of $H b$. salinarum PHH1 are only formed by pGvpA: the pGvpE activator protein produced from the p-vac region is unable to stimulate the expression of the c-gvpACNO gene cluster present in the same cell. The latter gvp gene cluster is only activated by the homologous cGvpE activator protein.

Archaeal promoters consist of a TATA box centred around position -28 relative to the transcriptional start site, and many promoters contain the TFB recognition element BRE (consensus: RNWAAW, $\mathrm{R}=\mathrm{A}$ or $\mathrm{G}, \mathrm{N}=$ any base, $\mathrm{W}=\mathrm{A}$ or $\mathrm{T}$ ) (Bell et al., 1999). The p-gvpA promoter contains a TATA box almost identical to the archaeal consensus, and also a highly conserved putative BRE element (Fig. 1a). These features could be the reason for the relatively high basal activity of the p-gvpA promoter, which does not depend on an activator protein for expression. In contrast, the inactive c-gvpA promoter shows less conservation in the TATA box, and no sequences reminiscent of a BRE element (Fig. 1a). Activation of this promoter required the cGvpE activator protein; neither $\mathrm{pGvpE}$ nor mcGvpE were sufficient for activation.

For a preliminary investigation of the DNA sequences required for GvpE stimulation, a chimeric pAcA promoter was tested for $b g a H$ expression in transformants. This promoter fragment consisted mainly of c-gvpA sequences, but $21 \mathrm{nt} 5^{\prime}$ to the TATA box were substituted by the respective sequences derived from the p$g v p A$ promoter, including the almost perfect BRE element (Fig. 1a). The pAcA-bgaH transformants indicated no basal $\beta$-galactosidase activity, suggesting that the putative BRE element next to the TATA box was not sufficient for transcription initiation at this promoter. However, the pAcA-bgaH construct was induced by all three GvpE proteins in the respective transformants, and cGvpE was the strongest activator. It is still not known where the GvpE activator protein(s) bind in the promoter region for activation, but the fact that all GvpE proteins were able to activate the pAcA-bgaH gene suggested that the sequences upstream of the TATA box were important in this process. Mutagenesis of the entire promoter region is in progress to determine overall promoter strength and the nucleotides important for the activation by GrpE.

\section{The $\beta$-galactosidase activity is more stable than the bgaH mRNA}

The investigation of the amount of $b g a H$ mRNA in the mcA-bgaH/cE transformant indicated a high amount of mRNA during the exponential and early stationary phases, and no bgaH mRNA in the late stationary phase. The increase in the $\beta$-galactosidase activity was in accordance with the increase in the mRNA during the exponential phase, but stayed at a high level during the stationary phase, implying that the $\beta$-galactosidase protein is rather stable. Thus, the bgaH reporter system is useful to determine the initial promoter activation.
However, since the $b g a H$ mRNA and $\beta$-galactosidase stabilities deviate from each other, promoter deactivation studies are not possible. This observation contrasts with results presented by Patenge et al. (2000), where the promoter of the bacterio-opsin (bop) gene and various $f d x$ promoter mutants were analysed with the $b g a H$ reporter system in the related species $H b$. salinarum. In the case of the bop promoter-bgaH fusion, the $\beta$-galactosidase activity remained at a high level for $96 \mathrm{~h}$, but was reduced in the single sample taken after $120 \mathrm{~h}$. However, other transformants contained stable amounts of $\beta$-galactosidase activity (Patenge et al., 2000).

Overall, the $b g a H$ reporter gene has turned out to be a useful tool for the investigation of promoter and regulatory activities at a more quantitative level. Although Hf. volcanii is only distantly related, halobacterial promoters derived from $H f$. mediterranei and $H b$. salinarum can be studied and compared to each other in this system. In further experiments, we hope to gain more insights into the activation of the various gvpA promoters by GvpE, and study the action of other regulatory proteins encoded by the vac region.

\section{ACKNOWLEDGEMENTS}

This work received financial support from the Deutsche Forschungsgemeinschaft (PF 165/6-3 and 165/8-1). We thank Mike Dyall-Smith for plasmid pMLH32 prior to publication, Richard Röder for plasmid mc-gvpE-pJAS, and Peter Zimmermann, Jobst Gmeiner and Kathryn Nixdorff for critical reading of this manuscript. Lovastatin was a generous gift of MSD Sharp \& Dohme GmbH (München).

\section{REFERENCES}

Ausubel, F. M., Brent, R., Kingston, R. E., Moore, D. D., Seidman, J. G., Smith, J. A. \& Struhl, K. (1988). Current Protocols in Molecular Biology, vol. 1. New York: Greene Publishing Associates and Wiley-Interscience.

Baglia, N., Goo, Y. A., Ng, W. V., Hood, L., Daniels, C. \& DaSarma, S. (2000). Is gene expression in Halobacterium NRC-1 regulated by multiple TBP and TFB transcription factors? Mol Microbiol 36, 1184-1185.

Bell, S., Jaxel, C., Nadal, M., Kosa, P. \& Jackson, S. (1998). Temperature, template topology, and factor requirements of archaeal transcription. Proc Natl Acad Sci U S A 95, 15218-15222.

Bell, S., Kosa, P., Sigler, P. \& Jackson, S. (1999). Orientation of the transcription preinitiation complex in Archaea. Proc Natl Acad Sci U S A 96, 13662-13667.

Chomczynski, P. \& Sacchi, N. (1987). Single step method of RNA isolation by acid guanidinium thiocyanate-phenol-chloroform extraction. Anal Biochem 162, 156-159.

Cline, S. W., Schalkwyk, L. C. \& Doolittle, W. F. (1989). Transformation of the archaebacterium Halobacterium volcanii with genomic DNA. J Bacteriol 171, 4987-4991.

DasSarma, S., Arora, P., Lin, F., Molinari, E. \& Yin, L. (1994). Wildtype gas vesicle formation requires at least ten genes in the gvp gene cluster of Halobacterium halobium plasmid pNRC100. J Bacteriol 176, 7646-7652.

Ellenberger, T. E., Brandl, C. J., Struhl, K. \& Harrison, S. C. (1992). The GCN4 basic region leucine zipper binds DNA as a dimer of 
uninterrupted $\alpha$ helices: crystal structure of the protein-DNA complex. Cell 71, 1223-1237.

Englert, C., Horne, M. \& Pfeifer, F. (1990). Expression of the major gas vesicle protein in the halophilic archaebacterium Haloferax mediterranei is modulated by salt. Mol Gen Genet 222, 225-232.

Englert, C., Krüger, K., Offner, S. \& Pfeifer, F. (1992a). Three different but related gene clusters encoding gas vesicles in halophilic archaea. J Mol Biol 227, 586-592.

Englert, C., Wanner, G. \& Pfeifer, F. (1992b). Functional analysis of the gas-vesicle gene cluster of the halophilic archaeon Haloferax mediterranei defines the vac-region boundary and suggests a regulatory role for the gvpD gene or its product. Mol Microbiol 6, 3543-3550.

Hausner, W., Wettach, J., Hethke, C. \& Thomm, M. (1996). Two transcription factors related with the eucaryal transcription factors TATA-binding protein and transcription factor IIB direct promoter recognition by an archaeal RNA polymerase. J Biol Chem 271, 30144-30148.

Holmes, M. L. \& Dyall-Smith, M. (2000). Sequence and expression of a halobacterial $\beta$-galactosidase gene. Mol Microbiol 36, 114-122.

Holmes, M. L., Nuttall, S. D. \& Dyall-Smith, M. (1991). Construction and use of halobacterial shuttle vectors and further studies on Haloferax DNA gyrase. J Bacteriol 12, 3807-3813.

Holmes, M. L., Scopes, R., Moritz, R., Simpson, R., Englert, C., Pfeifer, F. \& Dyall-Smith, M. (1997). Purification and analysis of an extremely halophilic $\beta$-galactosidase from Haloferax alicantei. Biochim Biophys Acta 1337, 276-286.

Horne, M. \& Pfeifer, F. (1989). Expression of two gas vacuole protein genes in Halobacterium halobium and other related species. Mol Gen Genet 218, 437-444.

Horne, M., Englert, C., Wimmer, C. \& Pfeifer, F. (1991). A DNA region of $9 \mathrm{~kb}$ contains all genes necessary for gas vesicle synthesis in halophilic archaebacteria. Mol Microbiol 5, 1159-1174.

Krüger, K. \& Pfeifer, F. (1996). Transcript analysis of the c-vac region, and differential synthesis of the two regulatory gas-vesicle proteins GvpD and GvpE in Halobacterium salinarium PHH4. J Bacteriol 178, 4012-4019.

Krüger, K., Hermann, T., Armbruster, V. \& Pfeifer, F. (1998). The transcriptional activator GvpE for the halobacterial gas vesicle genes resembles a basic region leucine-zipper regulatory protein. J Mol Biol 279, 761-771.

Lam, W. L. \& Doolittle, W. F. (1989). Shuttle vectors for the archaebacterium Halobacterium volcanii. Proc Natl Acad Sci U S A 86, 5478-5482.

Ng, W. L., Ciufo, S., Smith, T. \& 9 other authors (1998). Snapshot of a large dynamic replicon in a halophilic archaeon: megaplasmid or minichromosome? Genome Res 8, 1131-1141.

Ng, W. L., Kenney, S., Mahairas, G. \& 14 other authors (2000). Genome sequence of Halobacterium species NRC-1. Proc Natl Acad Sci U S A 97, 12176-12181.

Offner, S., Wanner, G. \& Pfeifer, F. (1996). Functional studies of the gvpACNO operon of Halobacterium salinarium reveal that the GvpC protein shapes gas vesicles. J Bacteriol 178, 2071-2078.
Offner, S., Ziese, U., Wanner, G., Typke, D. \& Pfeifer, F. (1998). Structural characteristics of halobacterial gas vesicles. Microbiology 144, 1331-1342.

Palmer, B. \& Marinus, M. (1994). The $d a m$ and $d \mathrm{~cm}$ strains of Escherichia coli-a review. Gene 143, 1-12.

Patenge, N., Haase, A., Bolhuis, H. \& Oesterhelt, D. (2000). The gene for a halophilic $\beta$-galactosidase ( $\mathrm{ggaH}$ ) of Haloferax alicante $i$ as a reporter gene for promoter analyses in Halobacterium salinarum. Mol Microbiol 36, 102-113.

Pfeifer, F. \& Blaseio, U. (1989). Insertion elements and deletion formation in a halophilic archaebacterium. J Bacteriol 171, 5135-5140.

Pfeifer, F. \& Ghahraman, P. (1993). Plasmid pHH1 of Halobacterium salinarium: characterization of the replicon region, the gas-vesicle gene cluster and insertion elements. Mol Gen Genet 238, 193-200.

Pfeifer, F., Offner, S., Krüger, K., Ghahraman, P. \& Englert, C. (1994). Transformation of halophilic archaea and investigation of gas-vesicle synthesis. Syst Appl Microbiol 16, 569-577.

Pfeifer, F., Zotzel, J., Kurenbach, B., Röder, R. \& Zimmermann, P. (2001). A p-loop motif and two basic regions in the regulatory protein GvpD are important for the repression of gas vesicle formation in the archaeon Haloferax mediterranei. Microbiology $147,63-73$.

Qureshi, S. \& Jackson, S. (1998). Sequence-specific DNA binding by the $S$. shibatae TFIIB homolog, TFB, and its effect on promoter strength. Mol Cell 1, 389-400.

Qureshi, S., Baumann, P., Rowlands, T., Khoo, B. \& Jackson, S. (1995). Cloning and functional analysis of the TATA binding protein from Sulfolobus shibatae. Nucleic Acids Res 23, 1775-1781.

Reeve, J. N., Sandman, K. \& Daniels, C. (1997). Archaeal histones, nucleosomes, and transcription initiation. Cell 89, 999-1002.

Röder, R. \& Pfeifer, F. (1996). Influence of salt on the transcription of the gas-vesicle genes of Haloferax mediterranei and identification of the endogenous transcriptional activator gene. Microbiology 142, 1715-1723.

Sambrook, J., Fritsch, E. F. \& Maniatis, T. (1989). Molecular Cloning: a Laboratory Manual, 2nd edn. Cold Spring Harbor, NY : Cold Spring Harbor Laboratory.

Thomm, M. (1996). Archaeal transcription factors and their role in transcription initiation. FEMS Microbiol Rev 18, 159-171.

Thompson, D. K., Palmer, J. R. \& Daniels, C. J. (1999). Expression and heat-responsive regulation of a TFIIB homologue from the archaeon Haloferax volcanii. Mol Microbiol 33, 1081-1092.

Zillig, W., Palm, P., Klenk, H.-P., Langer, D., Hüdepohl, U., Hain, J., Lanzendörfer, M. \& Holz, I. (1993). Transcription in archaea. In The Biochemistry of Archaea, pp. 367-391. Edited by M. Kates. Amsterdam: Elsevier Science.

Received 18 January 2001; revised 15 March 2001; accepted 19 March 2001. 